\title{
Delaunay-based Vector Segmentation of Volumetric Medical Images
}

\author{
Michal Španěl \\ Department of Computer Graphics and Multimedia \\ Faculty of Information Technology \\ Brno University of Technology \\ Božetěchova 2, 61266 Brno, Czech Republic \\ spanelefit.vutbr.cz
}

\begin{abstract}
Image segmentation plays an important role in medical image analysis. In this paper, a novel segmentation technique based on the 3D Delaunay triangulation is presented. A modified variational tetrahedral meshing approach is used to adapt a tetrahedral mesh to the underlying CT volumetric data so that image edges are well approximated in the mesh. In order to classify tetrahedra into regions/tissues whose characteristics are similar, three different clustering schemes are proposed. Finally, several methods for improving quality of the mesh and its adaptation to the image structure are also discussed.
\end{abstract}

\section{Categories and Subject Descriptors}

I.3 [Computer Graphics]: Computational Geometry and Object Modeling; I.4 [Image Processing and Computer Vision]: Segmentation

\section{Keywords}

Medical imaging, computed tomography, volumetric data, image segmentation, surface reconstruction, Delaunay triangulation, variational tetrahedral meshing, clustering.

\section{Introduction}

Medical imaging devices like the Computed Tomography (CT) and Magnetic Resonance (MRI) can be used to inspect patient body from the inside. These imaging devices produce image data detailing human anatomy. The medical image data can be used to create three-dimensional surface models of human anatomy. The advantage of a surface representation is that it gives a three-dimensional

\footnotetext{
* Recommended by thesis supervisor:

Assoc. Prof. Přemysl Kršek

Defended at Faculty of Information Technology, Brno University of Technology on January 5, 2011.

(C) Copyright 2011. All rights reserved. Permission to make digital or hard copies of part or all of this work for personal or classroom use is granted without fee provided that copies are not made or distributed for profit or commercial advantage and that copies show this notice on the first page or initial screen of a display along with the full citation. Copyrights for components of this work owned by others than ACM must be honored. Abstracting with credit is permitted. To copy otherwise, to republish, to post on servers, to redistribute to lists, or to use any component of this work in other works requires prior specific permission and/or a fee. Permissions may be requested from STU Press, Vazovova 5, 81107 Bratislava, Slovakia.

Španěl, M. Delaunay-based Vector Segmentation of Volumetric Medical Images. Information Sciences and Technologies Bulletin of the ACM Slovakia, Vol. 3, No. 1 (2011) 9-20
}

view from any angle. This is an improvement over the traditional investigation of two-dimensional grayscale images.

Substantial step of many image understanding methods is a segmentation that divides an image into parts that correspond to tissues of particular types. The segmentation is one of the most important steps in the analysis of the medical image data. The precise segmentation is crucial for 3D modeling of tissues and anatomical structures. In relation to the geometric modeling of human tissues, all medical image segmentation algorithms can be classified into two groups:

- techniques based on raster segmentation - a pixel value in the segmented image denotes label of an image region, or particular tissue type;

- and vector-based segmentation - region boundaries, and perhaps the internal structure, are represented as a set of vector graphic primitives (i.e. lines, curves, polygons, etc.).

Raster-based Segmentation Techniques. Many 2D/3D segmentation algorithms can be found in the literature (Fuzzy C-means clustering [27], Hidden Markov Fields [22] Watershed transform [15], neural networks, etc.). In these cases, an algorithm such as Marching Cubes [21] is applied to reconstruct surfaces from the raster segmented data. Further, decimation and smoothing [30] of the model are required and may not be elementary. Applied smoothing and decimation methods may not shrink significant edges and corners and they must preserve volume of the original model.

Vector-based Segmentation. Most widely used vector segmentation methods are based on deformable models [38]. Numerous researchers have explored application of deformable surface models to volumetric medical images [4]. A deformable surface model capable of segmenting complex internal organs such as the cortex of the brain has been proposed [23]. There is also a second type of deformable models - the geometric models, best known is the Level-Set method [9].

In general, deformable models are robust against noise and boundary gaps. These models are also capable of 


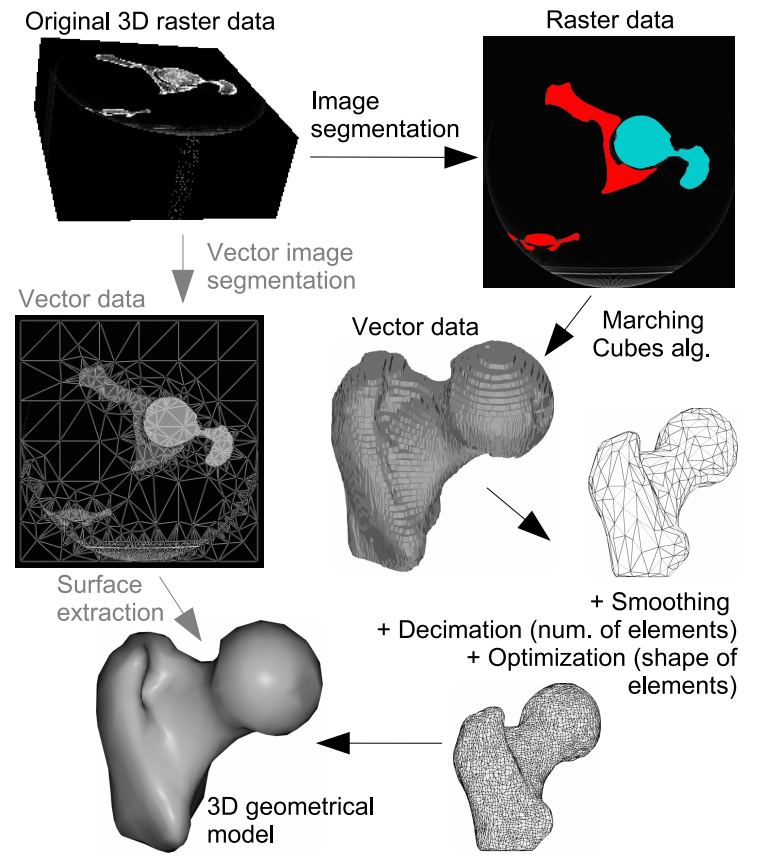

Figure 1: Comparison of the traditional rasterbased segmentation (black labeling) and the proposed vector segmentation method (underlined).

adjusting themselves to significant variability of human anatomy. Main disadvantage is that they require manual initialization and interaction during the segmentation.

\subsection{Unstructured Meshing}

A mesh generation [12] aims at tessellation of a bounded $3 \mathrm{D}$ domain $\Omega$ with tetrahedra. Algorithms for $3 \mathrm{D}$ mesh generation have been intensively studied over the last years. Basically, three main families of algorithms have been described:

- octree methods [39],

- advancing front methods [17],

- and Delunay-based methods $[6,1]$.

Zhang et al. [39] presented an algorithm to extract adaptive and quality $3 \mathrm{D}$ meshes directly from volumetric image data. In order to extract tetrahedral (or hexahedral) meshes, their approach combines bilateral and anisotropic diffusion filtering of the original data, with contour spectrum, iso-surface and interval volume selection.

The isosurface stuffing algorithm [19] was presented that fills an iso-surface with a uniformly sized tetrahedral mesh. The algorithm is fast, numerically robust, and easy to implement because, like the Marching Cubes, it generates tetrahedra from a small set of pre-computed stencils. A variant of the algorithm creates a mesh with internal grading. However, the algorithm does not permit grading of both surface and interior tetrahedra.

In this paper, a vector segmentation technique based on the variational tetrahedral meshing (VTM) approach, proposed by Alliez et al. [1], is presented. The VTM approach uses a simple quadratic energy to optimize vertex positions within the mesh and allow for global changes in mesh connectivity during energy minimization. This meshing algorithm allows to create graded meshes, and defines a sizing field prescribing the desired tetrahedra sizes within the domain.

\section{Tetrahedral Meshing}

Many applications have specific requirements on the size and shape of elements in the mesh. Aim of the isotropic meshing is to locate vertices so that the resulting mesh consists of almost regular tetrahedra $(\sim$ all faces are equilateral triangles). In addition, the element size is close to a predefined size constraint. One of the existing methods to create the points in accordance with the size specifications contained, creation of points along the edges [12], will be discussed here.

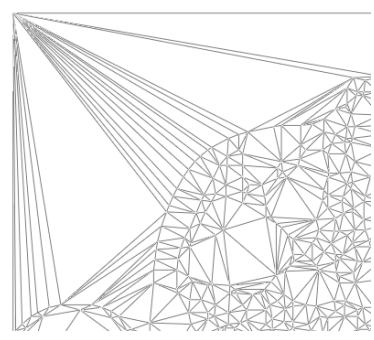

(a)

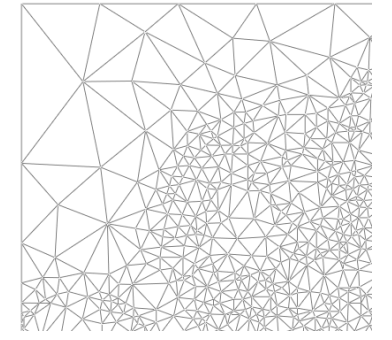

(b)
Figure 2: Triangular mesh constructed by the plain incremental method [12] (a) and result of the isotropic meshing (b).

According to [12], control space $H(\Omega)$ (so called sizing field) is a function $h_{P}$ defined at any point $P(x, y, z)$ of space. This function specifies the size of the elements in the mesh. Let $A B$ be an edge having endpoints $A$ and $B$. Length of the edge in the control space metric can be calculated as follows:

$$
l_{H}(A B)=\|A B\| \frac{\frac{1}{h(A)}+\frac{1}{h(B)}}{2},
$$

where $\|A B\|$ is the real distance between $A$ and $B$. The size $h(P)$ is the desired length of all the edges originating from the point $P$ defined by the control space. The key idea of the algorithm is to create new points along existing edges and obtain nearly regular tetrahedra having edges of unit length in the control space.

Construction of the points along the edge AB. Let $T$ be a threshold value $T<1$. If $l_{H}(A B)<T$, the edge is not divided, otherwise a new point in the middle of the edge $\mathrm{AB}$ is introduced. Both obtained sub-edges are recursively tested and divided if necessary, until we have a sequence of points $Q_{0} \ldots Q_{n}$ such that $l_{H}\left(Q_{i}, Q_{i+1}\right)<T$, where $Q_{0}=A$ and $Q_{n}=B$. The smallest index $i$ satisfying criterion $\sum_{j=0}^{i} l_{H}\left(Q_{j}, Q_{j+1}\right)>1$ is found and the point $Q_{i}$ is introduced to the mesh as new vertex. Iterating this process and comparing the sum to the increasing values $2,3, \ldots$ results in construction of several new points along the edge.

Applied to every edge in the current mesh, a large set of points is obtained. This set must be filtered to discard all 
a)

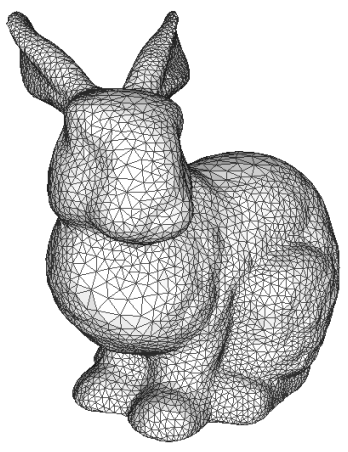

b)

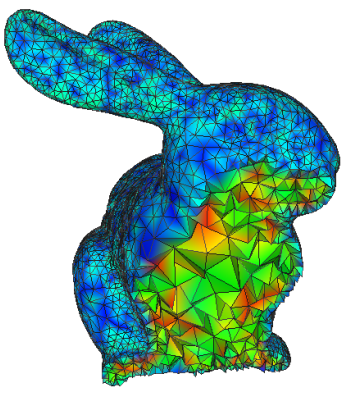

Figure 3: Results of the proposed vector segmentation method: surface extracted directly from the tetrahedral mesh (a); cut through the tetrahedral mesh (b).

points too close to any other before adding points to the mesh.

\subsection{Variational Meshing}

Many approaches based on energy minimization [10] have been proposed as a powerful tool in meshing. In this paper, a vector segmentation technique, built upon the VTM approach [1], is presented. A simple minimization procedure alternates two steps:

- global 3D Delaunay triangulation optimizing connectivity,

- and local vertex relocation,

to consistently and efficiently minimize a global energy over the domain. It results in a robust meshing technique that generates high quality meshes in terms of minimal dihedral angles.

To extend the approach to allow isotropic meshing, the sizing field $H$ is introduced. A mass density in space can be defined and used in computation of the optimal vertex position. This density should agree with the sizing field. Alliez et al. use a one-point approximation of the sizing field in a tetrahedron and defines the mass density as being $1 / h^{3}$ since the local volume of a tetrahedra should be roughly the cube of the ideal edge size. In geometric terms, the optimal position of the interior vertex $X_{i}$ in its 1-ring neighbourhood can be expressed as:

$$
X_{i}^{*}=\frac{1}{\sum_{T_{k} \in \Omega_{i}} \frac{\left|T_{k}\right|}{h^{3}\left(G_{k}\right)}} \sum_{T_{j} \in \Omega_{i}} \frac{\left|T_{j}\right|}{h^{3}\left(G_{j}\right)} c_{j} .
$$

where $G_{k}$ is the centroid of tetrahedron $T_{k}$ and $c_{j}$ is the circumcenter of tetrahedra $T_{j}$. Alliez et al. presented a default sizing field robust for a large spectrum of mesh types. Definition of the sizing field is built on the notion of local feature size that corresponds to the combination of domain boundary curvature and thickness as well.

Sizing Field. The local feature size $l f s(P)$ at a point $P$ of domain boundary is defined as the distance $d\left(P, S_{k}(\Omega)\right)$ to a medial axis $S_{k}(\Omega)$. The medial axis, or skeleton of the domain, is the locus of all centers of maximal balls inscribed in the boundary. Given the local feature size on the boundary, we need a controllable way to extrapolate this function to the interior. The function $h_{P}=\min _{S \in \delta \Omega}[K d(S, P)+l f s(S)]$ satisfies this criterion [1]. The parameter $K$ controls gradation of the resulting field, $K=0$ being the uniform case.

\section{Delaunay-based Vector Segmentation}

Based on the introduced principles, the Delaunay-based vector segmentation (shortly $\mathrm{VSeg}$ ) is proposed as follows:

1. Data preprocessing - Noise reduction by means of the $3 \mathrm{D}$ anisotropic (or bilateral filtering).

2. 3D edge and corner detection - Candidate vertices lying on region boundaries, meaningful edges and corners are located.

3. Initial Delaunay triangulation - Tetrahedral mesh is constructed from the sampled set of candidate vertices by the common Incremental method [12].

4. Iterative adaptation - The triangulation is adapted to the underlying image structure by means of isotropic edge splitting and variational meshing.

5. Mesh segmentation - Final classification of tetrahedra into image regions according to results of some data clustering method.

\subsection{Data Preprocessing}

An important part of the vector segmentation is adaptation of the tetrahedral mesh to an image structure which is derived from the found image edges. The adaptation process is strongly affected by quality of the edge detection. Therefore, it is highly recommended to filter the input data in order to deal with noise in the data.

In the preprocessing step, a 3D anisotropic filtering [26] is applied to the volumetric data. Gerig et al. [13] described an extension of the anisotropic filter into 3D space. Anisotropic (or bilateral [31]) filtering performs piecewise smoothing of the image, and its strength lies in the fact that object contours and boundaries are not only preserved, but even improved.

\subsection{D Edge and Corner Detection}

The triangulation starts from a set of candidate vertices distributed over the entire image. These candidates can be found by various image edge detection algorithms [5, $2,26]$ extended to $3 \mathrm{D}$ space.

Because of the complex nature of medical image data, detection of meaningful edges that form boundary of desired tissues may be very problematic. Character and strength of edges differ between tissues. Moreover, extremely thin and weak edges may be present in the image data. In practice, this leads to highly sensitive setting of the edge detector that, unfortunately, results in many false detections. In this paper, a simple tissue-selective edge detection approach is proposed to partially reduce this undesired effect. 


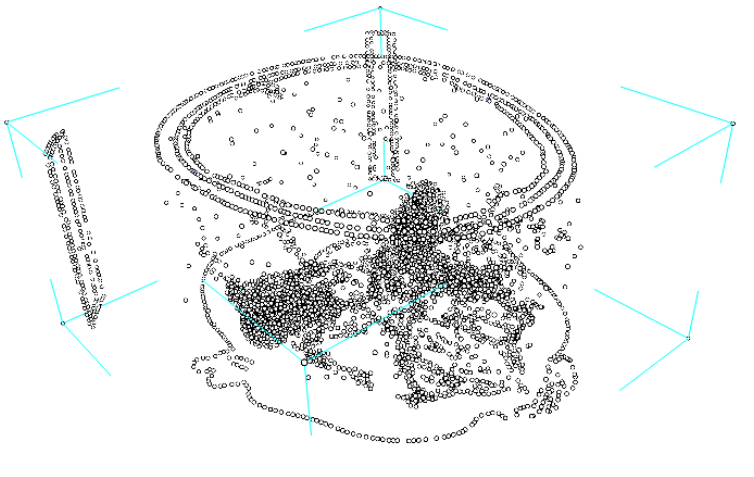

Figure 4: Sampled initial set of vertices found by the edge and corner detection.

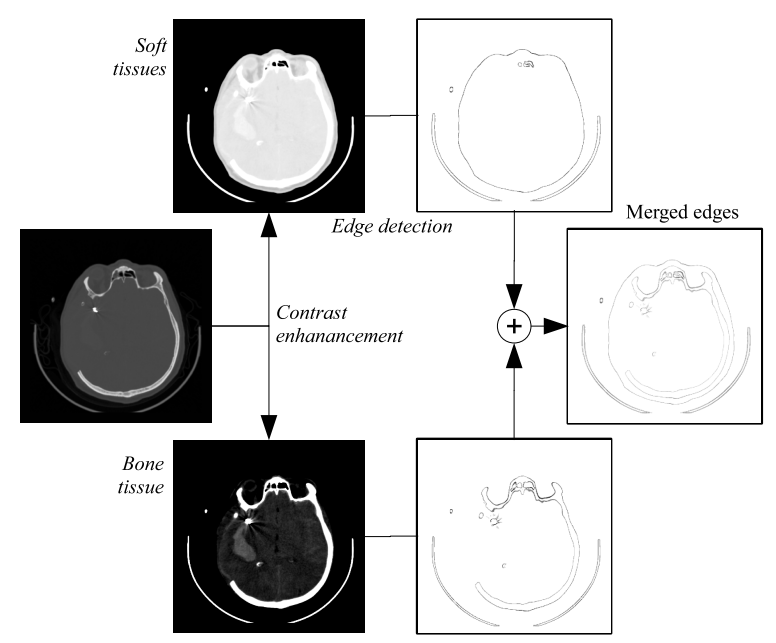

Figure 5: Scheme of the tissue-selective edge detection.

Tissue-Selective Edge Detection. The edge detection is divided into separate steps per concrete tissue type. Before the detection starts, the image data are pre-processed using the power-law contrast enhancement [14] technique to increase contrast of the desired tissue against all others. Then edges of the highlighted tissue are detected. In the end, all found edges from all different tissues are merged together into a single image (see Fig. 5). In our experiments, the well known Canny edge detector [5] extended to $3 \mathrm{D}$ space has been used in each step.

3D SUSAN Corner Detector. In order to respect significant features in the volumetric data during the meshing, we have modified the Susan corner detector [29] extending its functionality into 3D space. The Susan (Smallest Univalue Segment Assimilating Nucleus) detector was originally developed to locate feature points in 2D images. Analogous to Smith and Brady, the modified 3D SUSAN places a spherical mask $R$ over the voxel $v_{0}$ to be tested (the nucleus). Every voxel $v \in R$ is compared to the nucleus using the distance function $c_{v}$ Final response of the SUSAN detector [29] is proportional to $n(R)=\frac{1}{N} \sum_{v_{i} \in R} c_{v_{i}}$, where $N$ is the number of voxels within a spherical mask $R$ used as a normalization factor (see [29] for more details).

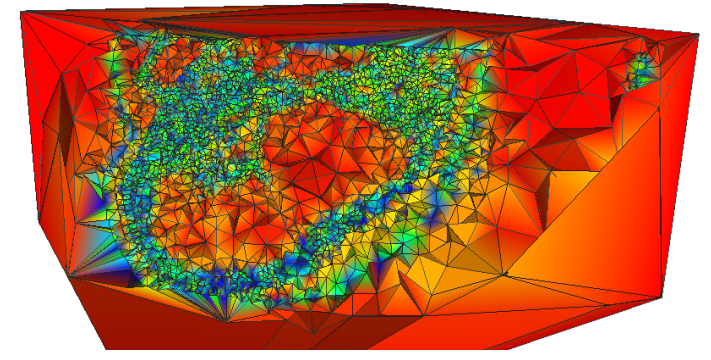

Figure 6: Final tetrahedral mesh after the iterative adaptation. Parameters of the sizing field were chosen to be $K=0.8$ and $T_{a v g}=70 \mathrm{~mm}$.

\subsection{Iterative Adaptation}

Fundamental phase of the proposed segmentation method is adaptation of the tessellation mesh to cover the underlying image structure. The following steps are repeated until the triangulation satisfies some convergence criterion (or just several times):

- Isotropic edge splitting - creation of points along existing edges introduces new points to the mesh,

- Variational meshing - optimization of the tessellation grid by means of vertex moving,

- Boundary refinement - creation of new vertices along image edges to guarantee that all edges are well approximated by the tessellation grid.

During the iterative adaptation, new vertices are gradually introduced to the mesh. The idea is to grow the mesh (in the sense of number of vertices) until a predefined limit is reached. An advantage of such progressive concept is that computational expensive operations like vertex removal are not necessary.

Before a new vertex is inserted to the mesh, several constraints are checked - minimal length of edges that will arise $\left(L_{m i n}\right)$, minimal dihedral angle inside newly created tetrahedra $\left(\alpha_{m i n}\right)$, etc. In practice, these constraints guarantee that chosen parameters like minimal edge length will be satisfied in the final mesh. Moreover, it prevents failures caused by a limited precision of math operations.

\subsubsection{Isotropic Edge Splitting}

In this phase, the isotropic meshing algorithm creating new points along existing edges and another well known technique of tetrahedral mesh optimization, splitting of maximal/longest edge [12], are combined together. Instead of maximal edges, those edges crossing significant image edges are divided. A new vertex is inserted to the mesh at the point of intersection of both edges. The whole isotropic edge splitting process can be briefly formulated as follows:

1. Sequentially process every edge $A B$ in the current triangulation $T^{i}$ :

- Find all intersection points $P_{i}$ of the edge and image edges. 
- Introduce the sub-edges $A P_{1}, P_{1} P_{2}, \ldots, P_{n} B$ in the triangulation.

- Divide all sub-edges in the sense of isotropic meshing algorithm (Sec. 2).

2. Filter the set of newly created points to discard vertices too close to any other point respecting the control space metric.

3. Insert points to the mesh $T^{i} \rightarrow T^{i+1}$.

To prevent degradation of the mesh, the angle between the tetrahedron $e_{t}$ edge and the image edge $e_{i}$ is computed. The splitting operation is performed only if the angle is greater than a given threshold $\alpha>10^{\circ}$. Edges that are almost parallel with an image edge remain unchanged.

Control Space. The control space, so called sizing field, prescribes length of edges in the mesh. In our case, the control space enforces creation of larger tetrahedra inside image regions and smaller ones along region boundaries (image edges). The control space $H(\Omega)$ can be defined in the same way as the sizing field given in Sec. 2.1. However, instead of the conventional domain boundary, we define the control space to respect found image edges. Thus, we generate the control space differently:

1. Estimate distance transform from all detected image edges first.

2. Find local maxima of the distance transform in order to identify medial axis.

3. Evaluate local feature size $\operatorname{lf} s(P)$ on image edges using inverse distance transform propagating value from the medial axis.

4. Generate control space distributing $\operatorname{lfs}(P)$ from edges.

This sizing field is relative. It describes the inhomogeneity of the required edge length. The real edge length is proportional to this relative value, and depending on the prescribed number of vertices. Such relative sizing is satisfactory for variational meshing, but it must be normalized for the isotropic edge splitting algorithm. The normalization is simply given by: $h_{P}^{\prime}=h_{P} T_{a v g} \frac{N}{\sum_{v \in \Omega} h_{v}}$, where $T_{a v g}$ is the desired average tetrahedron edge size. If the point $P$ lies exactly on an image edge, the control space value may be very small. Therefore, the minimal control space value $L_{\min }$ must be also specified in practice.

Setting smaller $K$ results in a visible gradation of the mesh not only inside the domain, but also on boundaries. The local feature size derived from medial axis takes more importance. As the $K$ increases, the gradation depends more on the distance from the boundary, the gradation affects only interior parts of the mesh.

\subsubsection{Variational Meshing}

The variational meshing phase, alternating connectivity and geometry optimization, is an important part of the algorithm. The mesh energy is minimized by moving each interior vertex to its optimal position within its 1-ring neighborhood (Fig. 9). Further, the energy is minimized

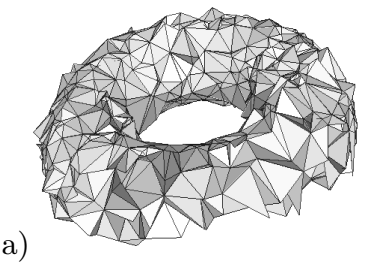

b)

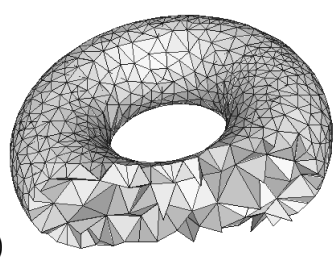

Figure 7: Variational tetrahedral meshing - the initial mesh (a); the same mesh after 3 iterations of the VTM algorithm (b).

by computing the 3D Delaunay triangulation of these new sites optimizing the connectivity of vertices.

All boundary vertices are treated differently. In order to identify the current boundary vertices, each voxel $V_{i}$ lying on an image edge is examined. Its nearest vertex $S_{j}$ in the mesh is located, and the distance $d\left(V_{i}, S_{j}\right)$ as well as the coordinates of $V_{i}$ (multiplied by the distance $d$ ) are accumulated at that vertex. To deal with corner points, the distance $d$ is weighted according to the point type. Corner points have the weight significantly greater than edge points, thus the closest vertex is attracted directly in place of the image corner. Afterwards, vertices with a non-zero distance sum are those boundary vertices we are looking for. These vertices are moved to the average value they each have accumulated during the pass over all edge voxels.

\subsubsection{Boundary Refinement}

The boundary refinement increases quality of the mesh in the sense of image edges approximation. Similarly to other Delaunay refinement methods, new vertices are added to the mesh to guarantee this criterion. In the first step, an algorithm similar to the identification of boundary vertices during the variational meshing is applied to locate proper places for new vertices:

1. Prepare an array of accumulators containing coordinates and distance of the edge point closest to each vertex. Initialize the distance to some large value $d_{\max }$.

2. For each voxel $V_{i}$ lying on an image edge:

(a) Locate its nearest vertex $S_{j}$.

(b) Compare the distance $d\left(V_{i}, S_{j}\right)$ with the value currently stored in the corresponding accumulator.

(c) If the distance is smaller, exchange the values in the accumulator.

In the second step, all accumulators that contain a distance lower than $d_{\max }$ are investigated. If there is a vertex with an accumulated value that is not itself located on an image edge, a new vertex is added to the mesh in place of the closest image edge point - the coordinates in the accumulator.

Dealing with Slivers. The variational meshing technique produces well shaped tetrahedra through the domain. Unfortunately, slivers could appear near the domain boundary. Recently, two powerful techniques $[20,32]$ capable of 


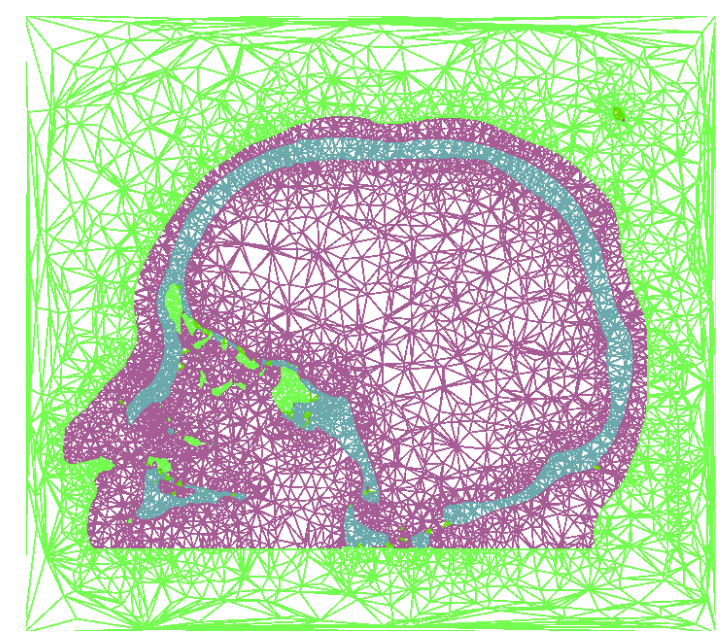

Figure 8: Result of the tetrahedral mesh segmentation phase - orthogonal cut through the classified mesh.

eliminating almost all slivers in the mesh were proposed. Both algorithms are based on explicit random perturbation of vertices incident to slivers.

Towards creation of a sliver-free mesh, after each iteration of the adaptation scheme, the mesh is repeatedly tested for slivers, and new vertices lying in the center of sliver circumspheres are inserted to the mesh with a small random perturbation. If such addition does not eliminate the sliver, or generates new one, the vertex position is perturbed again. Such vertex perturbation continues until an optimal position is found, thus the sliver can be successfully removed.

\subsection{Mesh Segmentation}

Within the mesh segmentation phase, all tetrahedra are classified into individual image regions. Every tetrahedron $t_{i}$ of the mesh is characterized by its feature vector. Individual features detail image structure of the tetrahedron, and perhaps its close neighborhood. Feature vectors may be grouped by the help of any conventional unsupervised clustering technique. Actually, three different algorithms were used for the unsupervised clustering of feature vectors into a certain number of classes:

- Fuzzy C-means (shortly FCM) algorithm [28],

- Gaussian Mixture Model optimized by the popular Expectation-Maximization (EM-GMM) algorithm [24].

- Min-Cut/Max-Flow graph-based algorithm [3].

First two techniques do not take into account any spatial/global information about the tetrahedra. Improvements can be made by incorporating global principles. Viewing the mesh as undirected graph allows to use graph algorithms (graph cuts, path-based clustering, etc.) for the segmentation. In this sense, the Min-Cut/Max-Flow [3] algorithm is used to cut a graph whose edges are evaluated according to a similarity of two adjacent tetrahedra.

The similarity of two adjacent tetrahedra (i.e. two feature vectors $P$ and $Q$ ) can be defined as a distance function in the feature space. Most common choice is the Manhattan or the Cosine distance function. An alternative is the use of criterions described in Sec. 3.4.1.

Feature Extraction. In fact, the first two components of a tetrahedron's feature vector are mean pixel value $\mu\left(t_{i}\right)$ and intensity variance $\sigma\left(t_{i}\right)$ of voxels inside the tetrahedron. Others may cover image texture/shape properties and spatial configuration of adjacent tetrahedra:

- features derived from gray level co-occurrence matrices [33],

- histogram of Local Binary Patterns (LBP) [25],

- wavelet features [34], etc.

The feature extraction is problematic if a tetrahedron is relatively small, just a few voxels are available. Simple grouping of adjacent tetrahedra into larger units may reduce this problem. Another solution is to reject classification of small tetrahedra, and use only relevant portion of the data. These non-classified tetrahedra, that appear mostly near to region boundary, will be assigned to particular regions in a next merging phase.

\subsubsection{Agglomerative Merging}

Topology of the tetrahedral mesh is suitable for image segmentation techniques such as region growing and merging. Instead of pixels and the traditional 4- and 8- pixel connectedness, tetrahedra adjacency is incorporated.

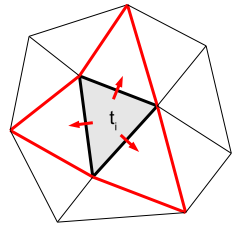

(a)

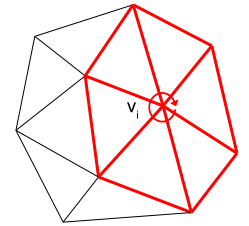

(b)

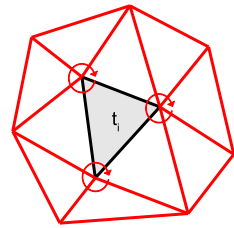

(c)
Figure 9: Tetrahedra adjacency (a); vertex 1-ring neighborhood (b); and tetrahedron 1-ring neighborhood.

In the vector segmentation scheme, the agglomerative region merging [18] is used to assign non-classified, small tetrahedra into already known segments. The agglomerative merging starts with a partition of the volumetric data into $\mathrm{N}$ regions (each region consists of one or more tetrahedra), and sequentially reduces the number of regions by merging the best pair of regions among all possible pairs in terms of a given criterion. This merging process is repeated until the required number of segments is obtained.

In practice, performance of this algorithm can be improved by a simple weighting of the similarity of two adjacent regions according to the number of voxels in both regions:

$$
C\left(r_{i}, r_{j}\right)=\frac{N_{i}+N_{j}}{N_{i} N_{j}} S\left(r_{i}, r_{j}\right)
$$




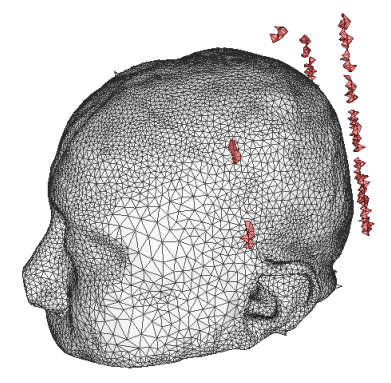

(a)

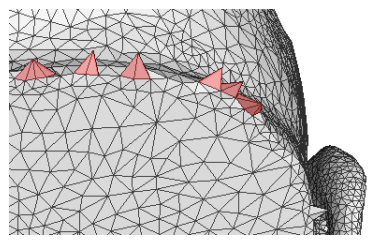

(b)
Figure 10: Surfaces extracted from the segmented mesh: an original surface without filtering of small isolated parts (a); artifacts that sometimes appear close to sharp edges and must be filtered (b).

If the final number of regions is unknown, the stopping criterion for the merging should be a ratio between similarities $C_{t-1}$ and $C_{t}$ of last two merged pairs of regions. To prevent early termination of the algorithm, approximately first $10 \%$ of all possible merges are made without any checking of this termination criterion.

Similarity Measures. Let $t_{i}$ and $t_{j}$ be two feature vectors extracted for a group of adjacent tetrahedra, or a single tetrahedron. Similarity measure is a function whose value is greater as the difference between two feature vectors decreases. Basic similarity measures are the mean intensity value and statistical test of the similarity based on voxel value variance:

$$
\begin{array}{r}
S_{\mu}\left(r_{j}, r_{i}\right)=\exp \left(-\frac{1}{2 \rho^{2}}\left|\mu_{r_{i}}-\mu_{r_{j}}\right|^{2}\right), \\
S_{\sigma}\left(r_{j}, r_{i}\right)=\frac{\sigma\left(r_{i}\right) \sigma\left(r_{j}\right)}{\sigma^{2}\left(r_{i, j}\right)},
\end{array}
$$

where the parameter $\rho$ affects sensitivity of the measure and $\sigma\left(r_{i}\right)$ is the variance of intensity in the region $r_{i}$ and $\sigma\left(r_{i, j}\right)$ is the variance of intensity in a joint region $r_{i} \cup r_{j}$.

Due to noisy input data and classification errors, some isolated tetrahedra, classified to a region different from its neighbors, may appear in the segmented mesh. This kind of misclassification can be reduced using a simple filtering scheme similar to the median filtering [14].

\subsubsection{Surface Extraction}

Once the mesh is properly segmented, surface of any region $R_{k}$ can be easily extracted. All tetrahedra through the mesh are traversed looking for boundary faces. The boundary faces can be intuitively identified as faces between two different regions. The extracted surface is closed and its mosaic conforms to the chosen parameters of the meshing - minimal required edge length $L_{\min }$, coefficient $K$ that controls gradation of the mesh, and the desired average tetrahedron size $T_{\text {avg }}$.

After the extraction, small isolated parts of the surface may be filtered to obtain a single closed surface if required. Moreover, to avoid artifacts that rarely appear on the surface, the final surface can be filtered for sharp spikes (Fig. 10).

\section{Experimental Results}

The vector segmentation was mainly designed for segmentation of volumetric medical images towards anatomical modeling of fundamental tissues (i.e. soft and bone tissue) and their surfaces. In order to evaluate precision, advantages and disadvantages of this method, number of experiments on real medical CT data, as well as on artificial volumetric data, were carried out. However, due to the complexity and variability of medical images, the testing was divided into several separate tasks.

\subsection{Surface Accuracy}

In case of the anatomical modeling, an error between reconstructed surfaces of human tissues and a "ground truth" must be minimal to guarantee correctness of a planned surgery. The following evaluation compares surfaces produced by the vector segmentation algorithm against ones made by the traditional Marching Cubes (MC) method followed by mesh smoothing and mesh decimation steps. Since the smoothing is crucial for overall precision of the surface, two standard approaches were tested:

- Taubin's smoothing algorithm [30] that maintains the volume of the mesh $(M C+$ Taubin $)$,

- HC algorithm [37] that preserves sharp edges and corners in the mesh $(M C+H C)$.

The MC algorithm produces very large meshes. Hence, after the smoothing, the Quadric Edge Collapse decimation algorithm, a variant of the well known edge collapse algorithm based on quadric error metric proposed by Michael Garland and Paul Heckbert [11], was used to reduce size of the mesh - the number of triangles. This re-meshing technique and both the utilized smoothing methods are implemented in the MeshLab [8] tool.

Artificial Data. Artificial volumetric data of basic solids such as rectangular solid, cylinder, cone, semi-sphere, pyramid and the Stanford bunny were generated for the testing. An idea of this measurement is to rasterize a solid into 3D raster, reconstruct surfaces from obtained artificial volumetric data, and evaluate an error between the reconstruction and the original surface.

The error between reconstructed surface and the original model was estimated using the Metro [7] tool. The Metro allows to compare the difference between a pair of surfaces (e.g. a triangulated mesh and its simplified representation) by adopting a surface sampling approach and point-to-surface distance computation. The approximation error between two meshes is defined as the distance between corresponding sections of the meshes. The mean distance and the maximum distance between the two meshes are presented as measures of the surface accuracy.

Fig. 11 shows histograms of error distribution for surface models of different level of detail as returned by the Metro tool. Apparently, the VSeg method outperforms both the smoothing-based methods. However, the difference is more evident for smaller meshes. As the number of faces increases, the error distribution of the VSeg method moves towards the MC $+\mathrm{HC}$ method. Direct meshing of 


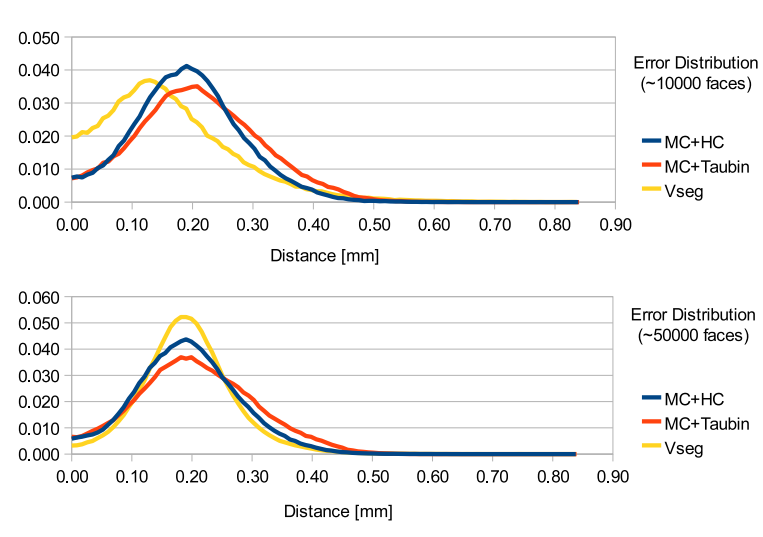

Figure 11: Histograms of the surface approximation error for two meshes with a different level of detail (number of faces) - the bunny model.
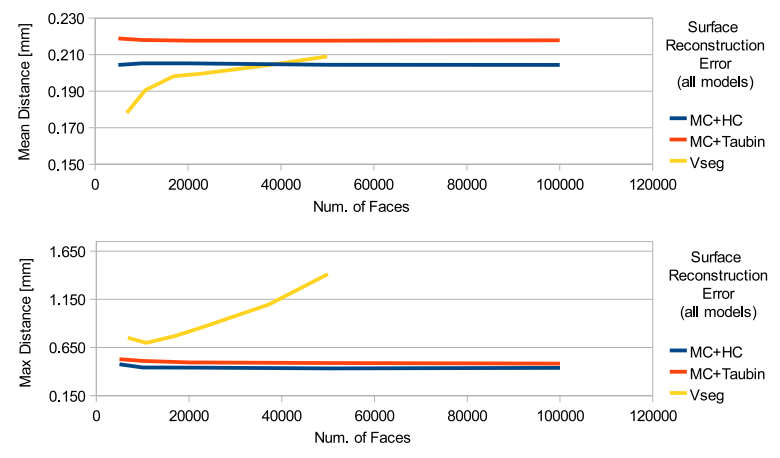

Figure 12: Overall surface approximation error whole set of test data.

volumetric image data seems to be more accurate approach then post-processing methods smoothing reconstructed surfaces without any relationship to the original image data.

Fig. 12 illustrates the overall mean approximation error and the maximal error depending on the number of faces in the mesh. The same behaviour as with the bunny model can be seen. The VSeg method outperforms the smoothing-based methods for smaller meshes up to $20 k$ faces. As the number of faces increases, the mean error of the VSeg method grows too. For meshes larger the $35 k$ faces, the surface approximation error exceeds the error of the $\mathrm{MC}+\mathrm{HC}$ method.

The question is why the performance decreases with the increasing number of faces? The answer lies in the iterative adaptation of the mesh to the underlying image structure. To obtain a more detailed surface, the minimal allowed edge length $L_{\min }$ must be decreased. However, the resolution of the raster data is limited. Decreasing the $L_{\min }$ down to the real size of a single voxel causes the relocation of vertices along image edges to not perform optimally. In the extreme case, the optimal vertex position is calculated as an average of a single "edge" voxel.

An interesting effect of the number of faces to the maximal error is notable in Fig. 12. In general, the maximal error, or maximal distance between sampled points

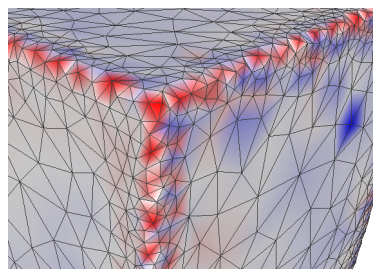

(a)

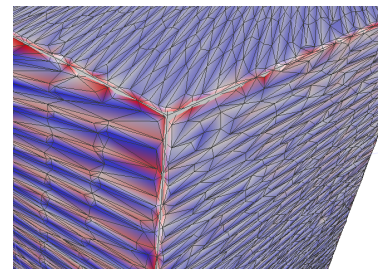

(b)
Figure 13: Error distribution on the reconstructed surface. The mean error of the VSeg method (a) over the entire surface is lower then the error of MC+HC method (b). On the other hand, the maximum error is larger along the sharp edges.
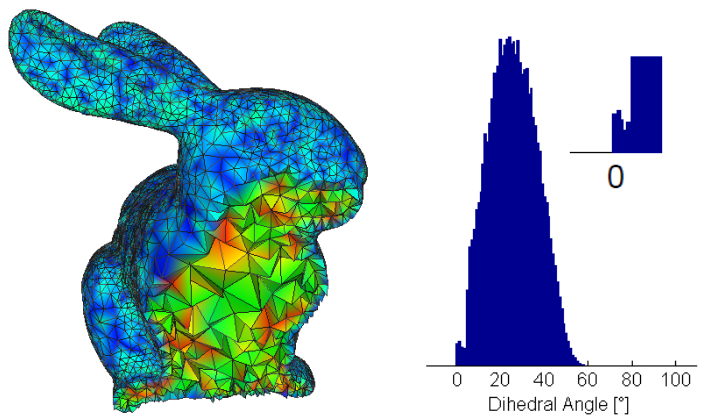

Figure 14: Cut through tetrahedral meshe colored according to the tetrahedra quality and histogram of minimal dihedral angles.

on compared surfaces, is much greater for surfaces obtained by the VSeg method than the ones produced by the MC-based approaches. The isotropic meshing generates high quality meshes with almost regular tetrahedra. Therefore, close to the sharp surface edges, the final mesh approximates the surface very roughly because of the limitation of tetrahedra shape and also the chosen minimal edge length (Fig. 13)

\subsection{Mesh Quality}

The proposed iterative mesh adaptation scheme was developed to produce high quality meshes suitable for many practical applications. Not only for the surface reconstruction of a desired tissue, but also for simulations that takes the internal structure of the tissue into account. The quality of produced meshes is discussed in this chapter.

Minimal Dihedral Angles. The original VTM approach produces well shaped tetrahedra inside the domain. However, poorly shaped tetrahedra and slivers may appear close to the boundary. Unfortunately, the same problem appears in case of the VSeg meshing method. Meshes in Fig. 14 are colored according to the quality of tetrahedra. Clearly, the quality of tetrahedra decreases as getting closer to the boundaries - the red shading moves towards blue. Even thought the embedded sliver elimination algorithm removes a large number of poorly shaped tetrahedra, it does not ensure that all slivers will be successfully eliminated. This is the reason why tetrahedra of a low quality $\left(\alpha_{\min }<3^{\circ}\right)$ are still present in all histograms. 

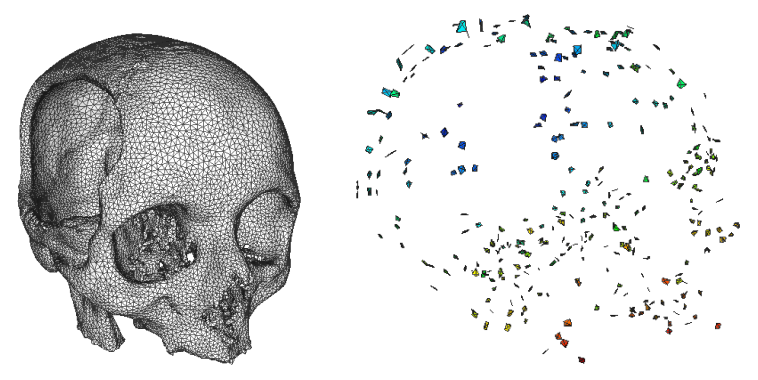

Figure 15: Illustration of slivers $\left(\alpha_{\min }<3^{\circ}\right)$ that still remain in tetrahedral meshes.

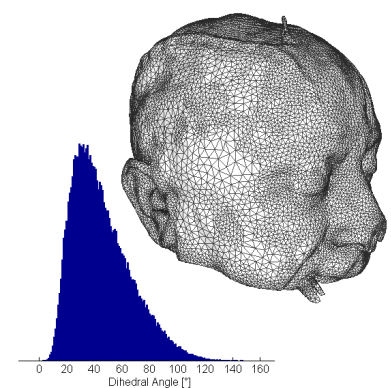

(a)

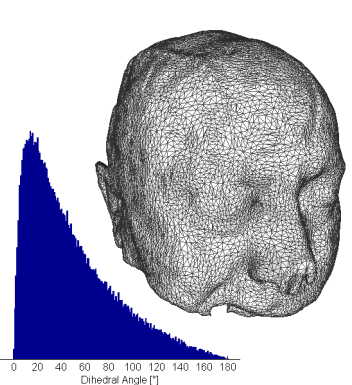

(b)
Figure 16: Quality of surfaces reconstructed using the VSeg method (a) and the MC+HC method. Histograms show distribution of dihedral angles.

One of the objectives was to provide meshes also suitable for numerical simulations. The presence of slivers may cause instability of such simulations. A smallest dihedral angle should be guaranteed. Recently, J. Tournois [32] has presented a new modification of the original VTM algorithm that particularly solves this problem and produces almost sliver free meshes.

\subsubsection{Surface Quality}

Quality of the extracted surface can be examined from two different sides. In the previous section, the approximation error of the surface has been studied. Here, the quality of surface with respect to the shape of triangles is briefly summarized.

Fig. 16 compares surfaces extracted from tetrahedral meshes (the VSeg method) against surfaces obtained from the $\mathrm{MC}+\mathrm{HC}$ method. Contrast between both methods is evident. The VSeg approach itself produces well shaped triangles along the entire surface, and moreover size of triangles is automatically adjusted according to a local complexity of the surface. Definitely, the MC meshes on the righthand side may be improved by existing re-meshing techniques, but such kind of post-processing increases the approximation error again.

\subsection{Mesh Segmentation}

The mesh segmentation phase was tested on several CT data sets clustering tetrahedra with respect to our requirements - surface reconstruction of main tissues (i.e. bones). The results were compared against manually annotated data - the ground truth.
During the segmentation, clustering techniques group only relevant, sufficiently large tetrahedra. Very small tetrahedra (fewer then 5-15 voxels) remain unclassified. These non-classified tetrahedra are assigned to particular regions in the subsequent merging phase (Sec. 3.4.1) which uses a linear combination of both presented simple similarity measures.

Ground Truth. Manual segmentation of medical images is a very complicated task. Not unfrequently, the segmentation made by different people varies. Every expert has his own view of the data and the correct segmentation. In order to quantify this phenomenon, one of the datasets were segmented by four different experts. Tab. 1 summarizes the obtained results.

Table 1: Difference between manual segmentations of the same dataset provided by four experts (the F-measure).

\begin{tabular}{c|cccc} 
Soft/Bone tis. & Man1 & Man2 & Man3 & Man4 \\
\hline Man1 & - & 0.921 & 0.965 & 0.896 \\
Man2 & 0.974 & - & 0.949 & 0.971 \\
Man3 & 0.993 & 0.979 & - & 0.922 \\
Man3 & 0.978 & 0.979 & 0.982 & - \\
\hline
\end{tabular}

An important issue is that the average error between two manual segmentations of the same data is about 0.96, measured by the F-measure of goodness which is described below. Occasionally, the error grows up (the F-measure decreases under) 0.92 .

F-measure. A way to match a segmentation to the ground truth is needed in order to evaluate performance of a segmentation algorithm. Many sophisticated measures of segmentation accuracy can be found in the literature [16]. An often used measure of segmentation goodness is the $F$-measure [35]. The F-measure combines recall $r$ and precision $p$ with an equal weight in the equation of the form:

$$
F_{\text {measure }}=\frac{2 r p}{r+p}, \quad p=\frac{T_{p}}{T_{p}+F_{p}}, \quad r=\frac{T_{p}}{T_{p}+F_{n}}
$$

where $p$ is the number of correctly labeled voxels (so called true positives $T_{p}$ ) divided by the total number of voxels labeled as belonging to the same region. The recall $r$ is defined as the number of true positives divided by the total number of elements that actually should belong to the positive class. A perfect score of the F-measure is 1, in the worst case the measure is equal to 0 .

\subsubsection{Meshing Segmented Data}

Without too much effort, the Delaunay-based segmentation can be applied to already segmented data. Fig. 17 shows an error between the obtained mesh and the segmented volumetric data. The F-measure rates how precisely the mesh approximates the original data. To compare this difference, all tetrahedra are rasterized into the volume data of the same size as the original one.

Results show that the error depends on initial setting of the meshing, it generally grows for meshes with larger 


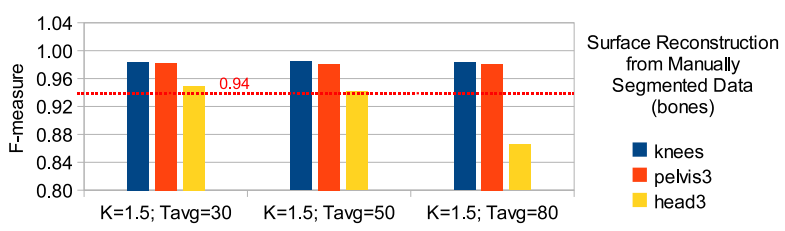

Figure 17: Surface reconstruction error when meshing pre-segmented data. The red line implies the error observed when several people labeled a same CT data set manually (see Tab. 1).

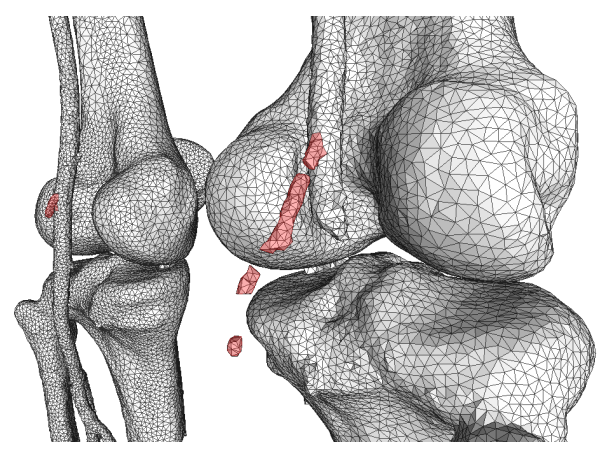

Figure 18: Surfaces reconstructed from presegmented data. In the red areas of the surface, small anatomical structures are weakly approximated because their size is relatively small compared to a prescribed minimal edge length.

tetrahedra. However, if adequate meshing parameters were chosen, the value is almost the same as the error, or variations, produced by different people when segmenting a same dataset (Tab. 1). In practice, large portion of this error is caused by limitations of the meshing process. All image structures smaller than the chosen minimal edge length $L_{\min }$ are lost. The mesh cannot approximate structures so small (Fig. 18).

\subsubsection{Segmentation of Medical CT Data}

In the last experiment, three different unsupervised clustering techniques (FCM, GMM+EM and Min-Cut/MaxFlow) were applied to meshes in order to classify tetrahedra into individual regions/segments. Fig. 19 recapitulates results of the mesh segmentation. All parameters of the meshing phase were experimentally set to optimal values (most often $K=1.5, T_{\text {avg }}=50$ and $L_{\min }=1.5$ ).

Results show that all clustering techniques are able to distinguish soft tissue. When compared to the manual segmentation, the VSeg method provides precise segmentation of the same quality as the voxel-based FCM clustering of the original image data. The segmentation error of soft tissues is comparable to the variation of manual segmentation of the same dataset by different individuals.

Not the worse results occur in case of bone segmentation from the head3 dataset. The VSeg method still produces quite good results. However, the measured error of the bone tissue segmentation significantly grows (i.e. value of the F-measure decreases) for the second dataset. Only the graph-based Min-Cut/Max-Flow algorithm provides rea-

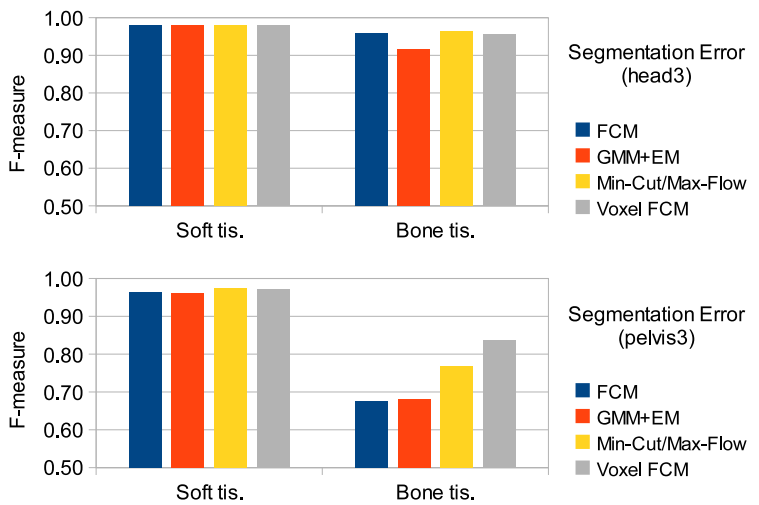

Figure 19: Segmentation error of the VSeg method - three alternative clustering methods are compared to the straight $F C M$ clustering of volumetric data (voxel FCM).

sonable results. Because the method takes spatial image structure more into account, results of this graph method overcomes other techniques.

Due to the thickness of the cortical bone and regarding resolution of CT data, very thin edges are present in the image data which are practically undetectable by conventional edge detection techniques without more knowledge of the data. Therefore, such kind of (non)edges is not well approximated during the meshing process which causes more errors in the final mesh segmentation. This nature of some medical CT data is also one of the reasons to allow manual corrections of the mesh segmentation.

\subsection{Runtime Statistics}

Basic runtime statistics can be found in Fig. 21. The measurement was divided into four stages: preprocessing of input data (i.e. anisotropic filtering), initialization of the meshing (the edge and corner detection; generation of the control space), iterative adaptation of the mesh, and the mesh segmentation. All phases take approximately 25 - 50 minutes on a standard PC with Intel Core2Duo $2.54 \mathrm{GHz}$ processor depending on a concrete size of the data and specific parameters of the meshing algorithm.

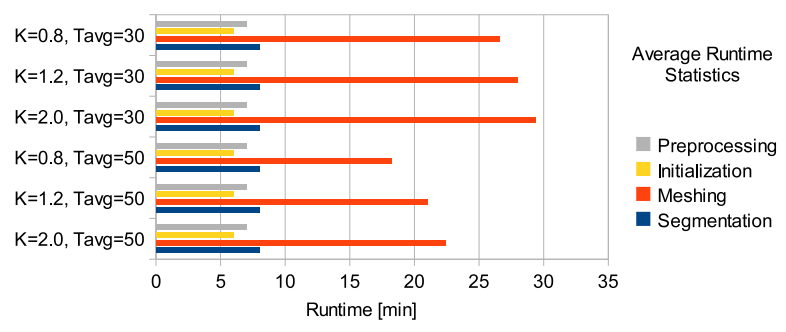

Figure 21: Runtime statistics of the VSeg method.

In fact the runtime of the vector segmentation is not very impressive. In comparison with traditional surface reconstruction techniques like the MC algorithm (+ subsequent smoothing), the VSeg method loses. These techniques are able to reconstruct surfaces in a much less time - just about minutes. However, such comparison is a bit unfair. Beside the surface, the VSeg method produces more com- 

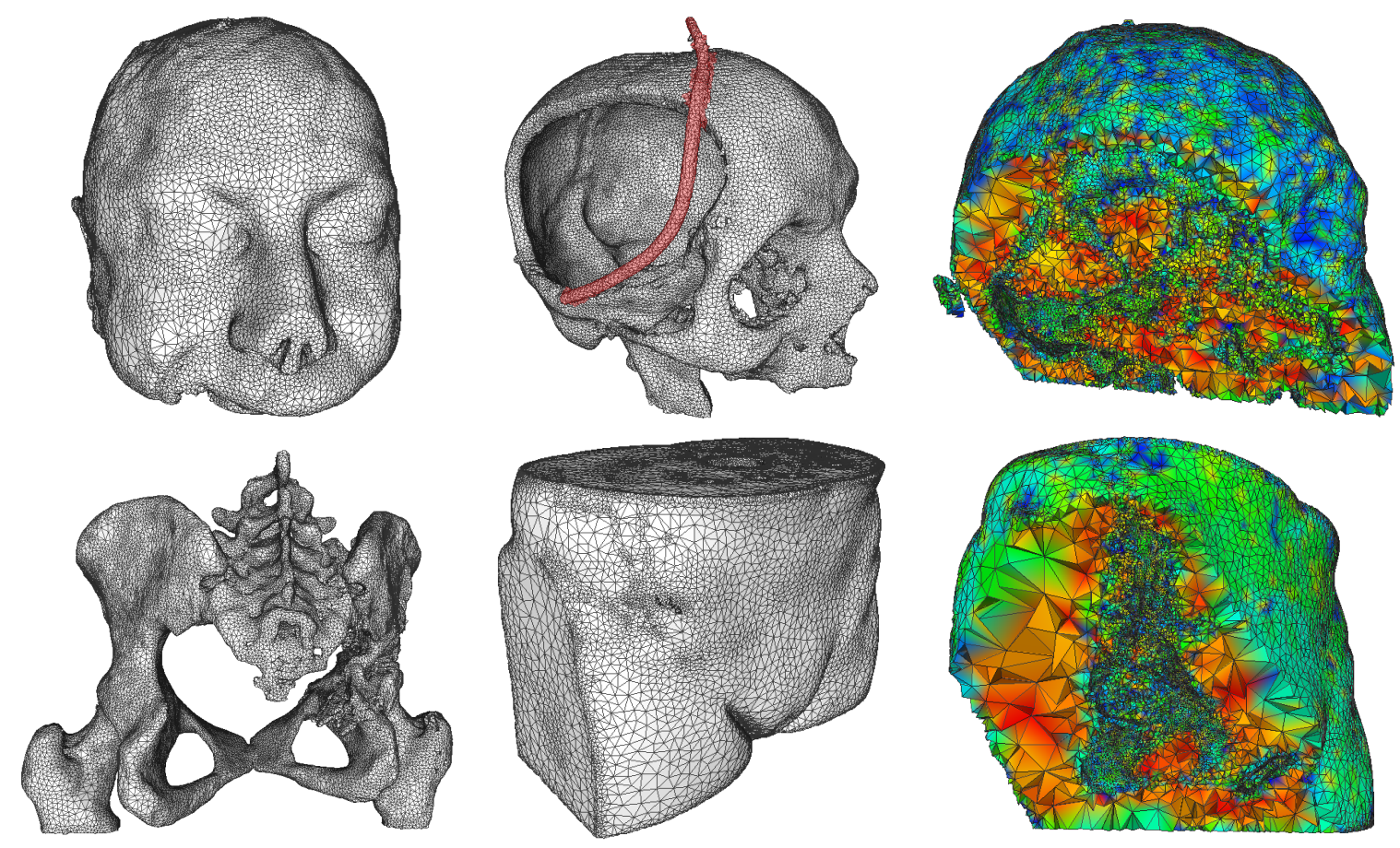

Figure 20: Surfaces and tetrahedral meshes extracted from CT data by the VSeg method. Meshing parameters were chosen $K=1.5, T_{a v g}=50 \mathrm{~mm}$, and $L_{\min }=1.5 \mathrm{~mm}$.

prehensive representation of the data - tetrahedral mesh - which may be useful for many other tasks. Aim of the thesis was to prove the concept of volumetric data segmentation based on Delaunay meshing. Not much attention was paid to the optimization of the implementation.

\section{Conclusions}

This paper presents a technique for segmentation of volumetric medical images aimed at surface reconstruction of fundamental human tissues (i.e. bone and soft tissues). This technique of vector segmentation is based on the 3D Delaunay triangulation. Tetrahedral mesh is used to partition the volumetric data. Such direct meshing of volumetric image data appears to be more accurate approach then traditional techniques which start with a surface extraction followed by the decimation and smoothing without any relationship to the original image data. Nevertheless, the idea of the vector segmentation has several other advantages.

A more effective representation of the image structure is obtained which approximates the original raster data. The mesh representation decreases complexity of the subsequent segmentation because of processing a reduced number of tetrahedra instead of a large number of voxels.

The proposed vector segmentation can be successfully used for surface reconstruction of desired tissues, as well as for meshing of the interior structure of the tissues for the numerical simulation. Obtained results show that the current concept works very well for certain CT data and is applicable to anatomical modeling of a human skull or soft tissues (i.e. craniectomy in case of traumatic brain injury). For the purpose of plain surface reconstruction from an already pre-segmented data, the VSeg method produces surfaces of more than reasonable quality and can be used as is.
However, several inconveniences can be still found in the method that are not very favourable from the practical point of view. Even thought the quality of reconstructed surfaces is sufficient for many applications, the quality of produced tetrahedral meshes is not as good as it could be. Slivers still appear close to region boundaries, and the method does not guarantee minimum dihedral angle in the mesh.

Another disadvantage can be found in the edge detection step which is crucial for precise approximation of image boundaries. The proposed tissue-selective edge detection works well for selected CT data. However, many parameters of the detection must be tuned to provide desirable results for other type of CT data. The edge detection limits potential application of the method in other research fields when different kind of volumetric data is used. Theses aspects of the proposed vector segmentation technique should be addressed in the future work.

\section{Acknowledgements.}

This work was partially supported by the BUT FIT grant FIT-S-10-2 and the research plan MSM0021630528. Many thanks to $O$. Veksler for his freely available implementation of the Min-Cut/Max-Flow algorithm [36] and thanks to 3Dim-Laboratory s.r.o. company for providing part of the test CT data.

\section{References}

[1] P. Alliez, D. Cohen-Steiner, M. Yvinec, and M. Desbrun. Variational tetrahedral meshing. ACM Trans. Graph., 24(3):617-625, 2005.

[2] M. Basu. Gaussian-based edge-detection methods: A survey. SMC-C, 32(3):252-260, August 2002.

[3] Y. Boykov and V. Kolmogorov. An experimental comparison of $\mathrm{min}$-cut/max-flow algorithms for energy minimization in vision. 
IEEE transactions on Pattern Analysis and Machine Intelligence, 26(9):1124-1137, September 2004.

[4] J. Bredno, T. M. Lehmann, and K. Spitzer. A general discrete contour model in two, three, and four dimensions for topology-adaptive multichannel segmentation. IEEE Trans. Pattern Anal. Mach. Intell., 25(5):550-563, 2003.

[5] J. Canny. A computational approach to edge detection. IEEE Trans. Pattern Anal. Mach. Intell., 8(6):679-698, 1986.

[6] P. R. Cavalcanti and U. T. Mello. Three-dimensional constrained delaunay triangulation: A minimalist approach. In Proceedings of the 8th International Meshing Roundtable, pages 119-129, Sandia National Laboratories, Lake Tahoe, California, USA, October 1999.

[7] P. Cignoni, C. Rocchini, and R. Scopigno. Metro: measuring error on simplified surfaces. In Computer Graphics Forum, volume 17, pages 167-174. Blackwell Publishers, 1998. http://vcg.sf.net/.

[8] V. C. L. I. CNR. Meshlab. http://meshlab.sourceforge.net/.

[9] M. Droske, B. Meyer, M. Rumpf, and C. Schaller. An adaptive level set method for medical image segmentation. In 17th International Conference on Information Processing in Medical Imaging, volume 2082, pages 416-422, London, UK, 2001. Springer-Verlag.

[10] G. Du and D. Wang. Tetrahedral mesh generation and optimization based on centroidal voronoi tessellations. Inter. Journal on Numerical Methods in Engineering, 56(9):1355-1373, 2003.

[11] M. Garland and P. S. Heckbert. Surface simplification using quadric error metrics. In SIGGRAPH '97: Proceedings of the 24th annual conference on Computer graphics and interactive techniques, pages 209-216, New York, NY, USA, 1997. ACM Press/Addison-Wesley Publishing Co.

[12] P.-L. George and H. Borouchaki, editors. Delaunay Triangulation and Meshing: Application to Finite Elements. Hermes Science Publications, Orlando, FL, USA, November 1998.

[13] G. Gerig, O. Kubler, R. Kikinis, and F. Jolesz. Nonlinear anisotropic filtering of mri data. Medical Imaging, IEEE Transactions on, 11(2):221 -232, jun 1992.

[14] R. C. Gonzalez and R. E. Woods. Digital Image Processing (3rd Edition). Prentice-Hall, Inc., Upper Saddle River, NJ, USA, 2006.

[15] V. Grau, A. U. J. Mewes, M. A. niz, R. Kikinis, and S. K. Warfield. Improved watershed transform for medical image segmentation using prior information. IEEE Transactions on Medical Imaging, 23(4):447-458, April 2004.

[16] Q. Huang and B. Dom. Quantitative methods of evaluating image segmentation. In ICIP '95: Proceedings of the 1995 International Conference on Image Processing (Vol. 3)-Volume 3, page 3053, Washington, DC, USA, 1995. IEEE Computer Society.

[17] Y. Ito, A. M. Shih, and B. K. Soni. Reliable isotropic tetrahedral mesh generation based on an advancing front method. In In Proceedings 13th International Meshing Roundtable. Williamsburg, VA, Sandia National Laboratories, pages 95-105, 2004.

[18] T. Kurita. An efficient agglomerative clustering algorithm for region growing. In Proc. of IAPR Workshop on Machine Vision Applications, Dec.1315, pages 210-213, 1991.

[19] F. Labelle and J. R. Shewchuk. Isosurface stuffing: fast tetrahedral meshes with good dihedral angles. ACM Trans. Graph., 26(3):57, 2007.

[20] X. Li. Sliver-free three dimensional delaunay mesh generation. Technical report, PH.D THESIS, UIUC, 2000.

[21] W. E. Lorensen and H. E. Cline. Marching cubes: A high resolution 3d surface construction algorithm. SIGGRAPH Comput. Graph., 21(4):163-169, 1987.

[22] J. L. Marroquin, E. A. Santana, and S. Botello. Hidden markoy measure field models for image segmentation. IEEE Transactions on Pattern Analysis and Machine Intelligence, 25(11):1380-1387, November 2003.

[23] T. McInerney and D. Terzopoulos. Medical image segmentation using topologically adaptable surfaces. In First Joint Conference of Computer Vision, Virtual Reality, and Robotics in Medicine and Medical Robotics and Computer-Assisted Surgery
(CVRMed-MRCAS'97), volume 1205, pages 23-32, Grenoble, France, 1997. Springer-Verlag.

[24] S.-K. Ng and G. J. McLachlan. On some variants of the em algorithm for fitting mixture models. Austrian Journal of Statistics, 23:143-161, 2003.

[25] T. Ojala, M. Pietikäinen, and T. Mäenpää. Multiresolution gray-scale and rotation invariant texture classification with local binary patterns. IEEE Transactions on Pattern Analysis and Machine Intelligence, 24(7):971-987, July 2002.

[26] P. Perona and J. Malik. Scale-space and edge detection using anisotropic diffusion. IEEE Transactions on Pattern Analysis and Machine Intelligence, 12:629-639, 1990.

[27] D. L. Pham. Robust fuzzy segmentation of magnetic resonance images. In 14th IEEE Symposium on Computer-Based Medical Systems, CBMS 2001, pages 127-131, Bethesda, MD, USA, July 2001.

[28] D. L. Pham and J. L. Prince. Adaptive fuzzy segmentation of magnetic resonance images. IEEE Transactions on Medical Imaging, 18, September 1999.

[29] S. M. Smith and J. M. Brady. Susan - a new approach to low level image processing. Inter. Journal of Computer Vision, 1996.

[30] G. Taubin and G. Taubin. Geometric signal processing on polygonal meshes, Sep 2000.

[31] C. Tomasi and R. Manduchi. Bilateral filtering for gray and color images. In ICCV '98: Proceedings of the Sixth International Conference on Computer Vision, page 839, Washington, DC, USA, 1998. IEEE Computer Society.

[32] J. Tournois, R. Srinivasan, and P. Alliez. Perturbing slivers in 3d delaunay meshes. In Proceedings of the 18th International Meshing Roundtable, pages 157-173. Springer Berlin Heidelberg, November 2009.

[33] F. Tsai, C.-K. Chang, J.-Y. Rau, T.-H. Lin, and G.-R. Liu. 3d computation of gray level co-occurrence in hyperspectral image cubes. In EMMCVPR'07: Proceedings of the 6th international conference on Energy minimization methods in computer vision and pattern recognition, pages 429-440, Berlin, Heidelberg, 2007. Springer-Verlag.

[34] M. Unser, A. Aldroubi, and A. Laine. Guest Editorial: Wavelets in Medical Imaging. IEEE Transactions on Medical Imaging, 22(3):285-288, 2003.

[35] C. J. van Rijsbergen. Information Retrieval 2nd Ed. Butterworths, London, 1979.

[36] O. Veksler. Gcmex - matlab wrapper for graph cuts multi-label energy minimization, 2010. http://vision.ucla.edu/ brian/gcmex.html.

[37] J. Vollmer, R. Mencl, and H. Mller. Improved laplacian smoothing of noisy surface meshes. In Computer Graphics Forum, volume 18, pages 131-138. Blackwell Publishing, Sep 1999.

[38] J. Zhang. Reconstruction of geometry from cardiac mr images. CVGIP: Image Understanding, 55:14-26, January 1992.

[39] Y. Zhang, C. Bajaj, and B.-S. Sohn. Adaptive and quality 3d meshing from imaging data. In SM '03: Proceedings of the eighth ACM symposium on Solid modeling and applications, pages 286-291, New York, NY, USA, 2003. ACM.

\section{Selected Papers by the Author}

M. Španěl, P. Kršek, M. Švub, V. Štancl. Vector Segmentation of Volumetric Image Data: Tetrahedral Meshing Constrained by Image Edges. In Proceedings of the 3rd International Joint Conference on Computer Vision, Imaging and Computer Graphics Theaory and Applications - GRAPP 2010, pp. 134-138, Portugal, 2010, INSTICC.

M. Švub, P. Kršek, M. Španěl, V. Štancl, R. Bartoň, J. Vad'ura. Feature preserving mesh smoothing algorithm based on local normal covariance. In Proceedings of WSCG'10, pages 6, Plzeň, CZ, ZČU v Plzni, 2010.

M. Španěl, P. Kršek, M. Švub, V. Štancl, O. Šiler. Delaunay-Based Vector Segmentation of Volumetric Medical Images. In Proceedings of the 12th International Conference on Computer Analysis of Images and Patterns - CAIP 2007, LNCS 4673, pp. 261-269, Berlin Heidelberg, DE, 2007, Springer. 